\title{
Tunable Erbium-Doped Fiber Lasers Using Various Inline Fiber Filters
}

\author{
Shien-Kuei Liaw ${ }^{1,4}$, Kuei-Chu Hsu' ${ }^{2}$, Nan-Kuang Chen ${ }^{3}$ \\ ${ }^{1}$ Department of Electronics Engineering, National Taiwan University of Science and Technology, Taiwan \\ ${ }^{2}$ Department of Optics and Photonics, National Central University, Taiwan \\ ${ }^{3}$ Department of Electro-Optical Engineering, National United University, Taiwan \\ ${ }^{4}$ Department of Mechanical Engineering, National Taiwan University, Taiwan \\ E-mail: skliaw@mail.ntust.edu.tw \\ Received February 2, 2010; revised March 23, 2010; accepted March 27, 2010
}

\begin{abstract}
Several high-performance and tunable erbium-doped fiber lasers are reviewed. They are constructed by using fiber Bragg gratings (FBGs) or short-wavelength-pass filters (SWPFs) as wavelength tunable components inside the laser cavity. Broadband wavelength tuning range including $\mathrm{C}$ - and/or S-band was achieved, and tunable laser output with high slope efficiency, high side-mode suppression ratio was obtained. These fiber lasers can find vast applications in lightwave transmission, optical test instrument, fiber-optic gyros, spectroscopy, material processing, biophotonic imaging, and fiber sensor technologies.
\end{abstract}

Keywords: Fiber Bragg Grating (FBG), Short-Wavelength-Pass Filter (SWPF), Tunable Fiber Laser, Optical Communication

\section{Introduction}

In recent years, fiber lasers have found a variety of applications in the testing of fiber components, fiber sensing and wavelength division multipling (WDM) systems, in which they are used to act as a backup source with ITU-T grids [1]. Also, fiber lasers are useful for spectroscopy, sensing protection, and fiber-optic gyro [2]. Partially because of their features, such as low wavelength sensitivity to temperature, low-intensity noise, and all-fiber construction, their advantages over non-fiber-based laser sources are potentially lowintensity noise, high output power, and compatibility with fiber components. Previous works have proposed design and/or characteristics valuation of fiber lasers, including multiple-ring cavity fiber laser [3], two separate erbium-doped fiber lasers [4], distributed feedback fiber lasers [5], and Brillouin erbium-doped fiber laser pumped using fiber Bragg grating (FBG) [6]. These fiber lasers, however, have fixed wavelengths that are not suitable for wavelength routing, reconfigurable switching and/or network protection. On the contrary, tunable erbium-doped fiber lasers could well fit such requirements. Nowadays, a variety of tunable fiber lasers have been demonstrated such as tunable single-frequency fiber lasers [7], coherent combining tunable lasers [8], tunable fiber-ring laser using bending effect [9], and so on.

In this paper, we overview several works regarding tunable fiber lasers done by our groups. The first kind is the FBG-based linear-cavity tunable fiber laser using an optical circulator (OC) [10], or a broadband fiber mirror (BFM) [11] as rear cavity end while the front cavity end is based on tunable FBGs (TFBGs) which could be tuned by applying strain. FBGs have become an enabling technology that provides convenient, cost-effective, and reliable solutions to a multitude of design problems in fiber module. Using a backward pump scheme at 1480 $\mathrm{nm}$ in [11], stable lasing output power of $21.14-\mathrm{mW}$ measured at $1544.8 \mathrm{~nm}$ was obtained with a threshold pump power of $8.0 \mathrm{~mW}$. A side-mode suppression ratio as high as $57 \mathrm{~dB}$, wavelength tuning range up to $30 \mathrm{~nm}$ with a step resolution of $0.5 \mathrm{~mm} / \mathrm{turn}$, and power variation less than $1.0 \mathrm{~dB}$ were achieved. The second kind is the short-wavelength-pass filter (SWPF)-based tunable fiber ring laser with lasing wavelength down to S-band using filters adopting a side-polishing [12] or fusedtapering [13] technique to attain the wavelength-dependent fundamental-mode cutoff concept. The fiber sidepolishing and fused-tapering techniques were both employed to fabricate wavelength tunable fiber filters. The laser can be tuned close to the short-wavelength edge of the available erbium gain bandwidth with tuning range of 
$26 \mathrm{~nm}$, signal-to-amplified-spontaneous-emission (ASE) ratio of around $40 \mathrm{~dB}$, and the full width half maximum (FWHM) linewidth of about $0.5 \mathrm{~nm}$. The single-longitude-mode (SLM) operation will be briefly discussed in Subsection 5.3. These two kinds of tunable in-line filterbased tunable fiber lasers as mentioned may broaden wavelength tuning range in either $\mathrm{C}$ - and/or S-band and will be addressed in detail. Both of them have graceful features of simple structure, compactness, ease in connection with fiber components, high-efficiency, and continuous-tuning, which make them promising for vast applications.

\section{Wavelength Tunable Mechanisms}

\subsection{Tunable Fiber Bragg Grating}

In principle, a wavelength shift in a FBG may be due to the changes in temperature, strain, pressure and/or other parameters. The shift in Bragg wavelength with strain and temperature can be expressed as [14]

$$
\begin{aligned}
& \Delta \lambda_{\mathrm{B}}= \\
& 2 n \Lambda\left(\left\{1-\left(\frac{n^{2}}{2}\right)\left[P_{12}-v\left(p_{11}+p_{12}\right)\right]\right\} \varepsilon+\left[\alpha+\frac{\left(\frac{\mathrm{d} n}{\mathrm{~d} T}\right)}{n}\right] \Delta T\right)(1)
\end{aligned}
$$

where $\varepsilon$ is the applied strain, $\Lambda$ is the period of fiber, $P_{i, j}$ are the Pockel's (piezo) coefficients of the stress-optic tensor, and $v$ is the Poisson's ratio. Note that $n$ is the effective refractive index of the fiber core as defined in Equation (1), $\alpha$ is the thermal expansion coefficient of the silica fiber with a typical value of $0.015 \mathrm{~nm} /{ }^{\circ} \mathrm{C}$, and $\Delta T$ is the temperature change in degree Celsius. The term $\left(n^{2} / 2\right)\left[P_{12}-v\left(P_{11}-P_{12}\right)\right]$ has a numerical value of 0.22 . The strain can be measured under a constant temperature according to the following equation:

$$
\frac{1}{\lambda_{\mathrm{B}}} \frac{\delta \lambda_{\mathrm{B}}}{\delta \varepsilon}=0.78 \times 10^{-6} \mu \varepsilon^{-1}
$$

where $\lambda_{\mathrm{B}}$ is the Bragg wavelength, and this value gives a "rule-of-thumb" measurement of wavelength shift for a FBG with strain of $1 \mathrm{~nm}$ per $1000 \mu \varepsilon$ at $1.31 \mu \mathrm{m}$. To design a strain tunable FBG, firstly, it is embedded in a strip of composite thermal plastic material and then is attached to L-shaped holders at both ends. The FBG is then mounted on a precision translational stage with a high-resolution micrometer. By strained or compressed tuning of the precise screw of the micrometer, we can apply both directions in the transverse displacement for increasing the tuning range up to $\pm 8 \mathrm{~nm}$. Two steel rods are attached to the sides of the FBG composite strip to confine the applied strain or stress to the longitudinal direction only. The micrometer has a resolution of 0.5 $\mathrm{mm} /$ turn and a full range of $5.0 \mathrm{~mm}$ in translational dis- tance, therefore up to ten turns can be applied to tune the FBG reflection wavelength.

The lasing wavelength as a function of turns of screw is shown in Figure 1(a), with a linear slope of $4.82 \mathrm{~nm} /$ $\mathrm{mm}$ translational distance. Thus, $\Delta \lambda_{\mathrm{B}}=0.00482 \mathrm{~nm} / \mu \mathrm{m} \times$ $d$, where $d$ is the displacement of the translational stage in unit of micrometers. Another way is to embed the FBG in the outer laminar. The composite with the TFBG embedded within is attached to a 3-point tuning device by using instant adhesive glue. By tuning the precision screw of the 3-point bending device either by straining or compressing, we can apply transverse displacement in either direction to easily attain a tunable range of $\pm 10 \mathrm{~nm}$. This eliminates any use of complicated or bulky components to perform the tuning function.

Figure 1(b) shows the superimposed tuning spectra of two homemade tunable FBGs. The demonstrated tuning range of each FBG is approximately $15 \mathrm{~nm}$ with reflectance of $99.9 \%$. Before tuning, FBG1 has a central reflection wavelength of $1540.5 \mathrm{~nm}$ while that of FBG2 is

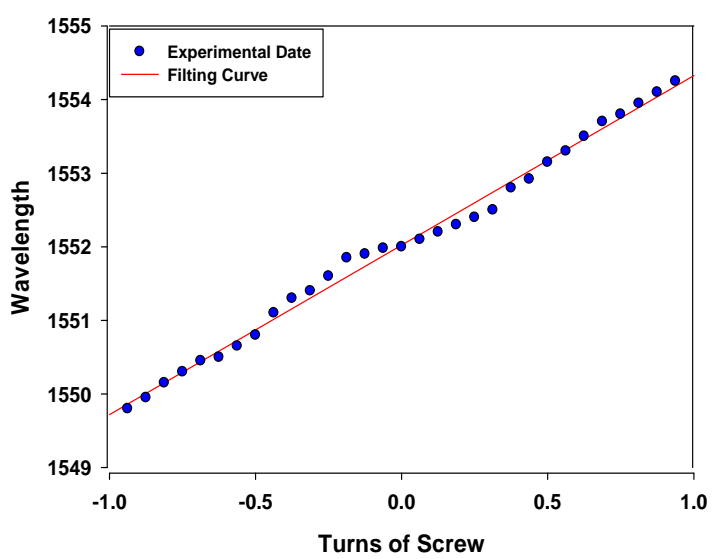

(a)

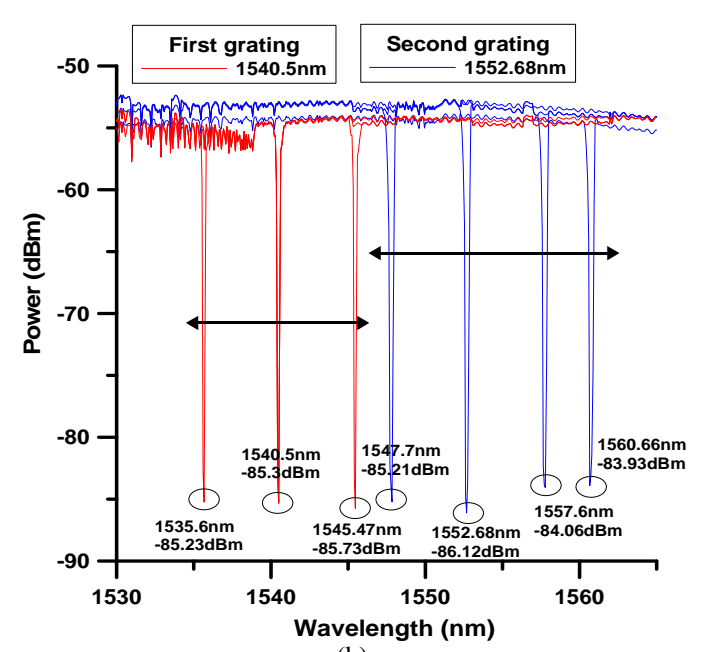

(b)

Figure 1. (a) Wavelength tunable FBG versus turns of micrometer screw; (b) superposed transmission spectra of two tunable FBGs (Total tuning range may cover the $C$ band). 
$1552.7 \mathrm{~nm}$. Fine tune resolution as precise as $0.2 \mathrm{~nm}$ FBG can be realized.

\subsection{SWPF-Based Tunable Fiber Laser}

The S-band tunable erbium-doped fiber lasers were achieved by connecting the active fiber to the thermooptic tunable SWPFs. The mechanism of the proposed SWPFs $[12,13]$ is to interact with the guiding optical fields to cause fundamental mode loss at long-wave length, and the cutoff wavelength can be tuned when the heating temperature applying on the filter changes. The dispersion engineering methods had been employed by controlling the propagation losses of lights at different wavelengths. Both the side-polishing and the fusedtapering techniques were adopted in our previous works $[12,13]$. When the SWPFs are temperature-tuned to attenuate the wavelengths longer than $1530 \mathrm{~nm}$, the $\mathrm{C}+\mathrm{L}$ band ASE is suppressed and the S-band gain is obtained. The commonly used S-band erbium-doped optical fiber amplifiers (EDFAs) employ erbium-doped fiber (EDF) with depressed inner cladding to achieve fundamental-mode cutoff at the longer wavelengths $[15,16]$. The cutoff wavelength and the mode field diameter can be adjusted through bending and local heating, but the fabrication, insertion loss, crosstalk and cost of the filters using dispersive fibers show great difficulties for practical use. Thus, an alternative way to obtain SWPFs is to interact with the light through the evanescent field that is spread out of the waveguide with wavelength-dependent properties. When the optical fiber is side-polished or tapered, the mode field is expanded out of the fiber cladding. Using dispersive liquids surrounding the side-polished fiber/taper fiber, the device can be a SWPF if the dispersion relations are properly designed. The tunable SWPF was achieved by tuning the temperature of the dispersive liquids to change the dispersive curves for obtaining different cutoff wavelengths $[17,18]$.

In Figure 2(a), the blue and red curves are the dispersion curves of the Ge-doped core and the fused silica of SMF-28, and the black curve is the dispersion for Cargille index-matching liquids. The refractive index dispersion of the core and cladding intersect at a fundamental mode cutoff wavelength which divides the wavelengths into bound and refracting leaky modes, and the lights with wavelength longer than the cutoff wavelength can be highly attenuated due to the frustrated total internal reflection. In our measurement, a broadband white light source was launched into the fabricated SWPF, where the optical liquid surrounding the SWPF was heated by a thermoelectric cooler (TEC) to stabilize the temperature. Figure 2(b) depicts the experimental and simulated transmission spectra of a SWPF over the tuning temperature of $25^{\circ} \mathrm{C}-27^{\circ} \mathrm{C}$, where the simulation results were performed by beam propagation method. The

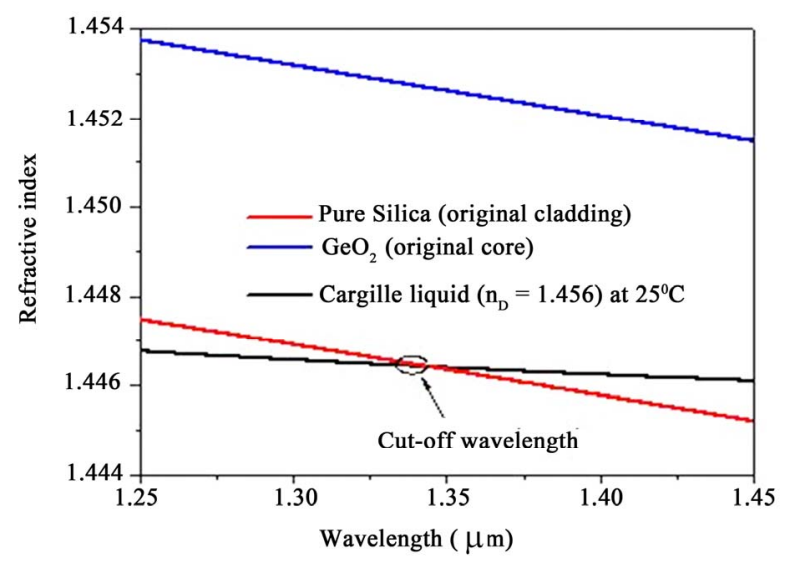

(a)

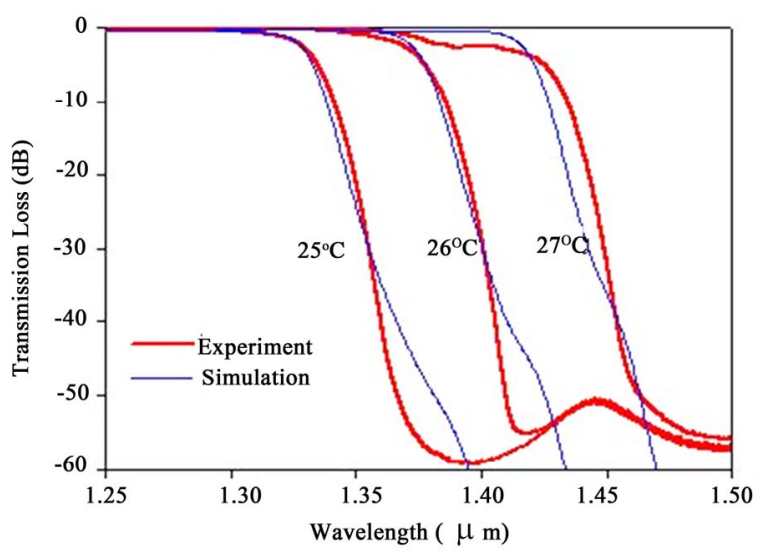

(b)

Figure 2. (a) Refractive index dispersion curves for index matching liquid and materials for original fiber core $\left(\mathrm{GeO}_{2}\right)$ and cladding (pure silica); (b) experimental and simulated spectral responses of tunable short-wavelength-pass fiber filter at different temperatures [18].

experimental results agree well with the simulation ones. The results include tuning efficiency of $50 \mathrm{~nm} /{ }^{\circ} \mathrm{C}$, cutoff efficiency of $-1.2 \mathrm{~dB} / \mathrm{nm}$, and rejection efficiency of $55 \mathrm{~dB}$. Based on these experimental and simulated results, the mechanism of the SWPF is proved to be qualified for developing wavelength tunable S-band erbiumdoped fiber lasers.

\section{FBG-Based Tunable Fiber Lasers: Configurations and Experimental/ Simulation Results}

\subsection{Optical Circulator as Laser's Rear Cavity End}

The proposed configuration of linear cavity for the tunable laser is shown in Figure 3. The linear cavity consists of a 3-port OC, two TFBGs, a segment of high- 


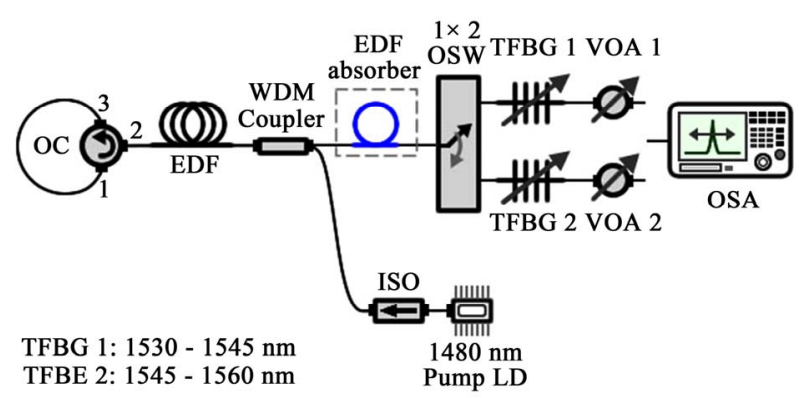

Figure 3. Configuration of proposed OC-based linear-cavity tunable fiber laser [10] (ISO: optical isolator; LD: laser diode; OSA: optical spectrum analyzer).

concentration EDF, a 1480/1550 nm WDM coupler, and one $1480 \mathrm{~nm}$ pumping source. The 3-port OC here acts as a wavelength router by connecting port 3 with port 1 . In this way, the residual pumping power travels back to the EDF for twice amplification to increase its pumping efficiency up to $2 \mathrm{~dB}$ difference in laser output power. A piece of EDF is inserted into the cavity to act as pump absorber. At the right hand side of this cavity, there is one $1 \times 2$ optical switch (OSW) and two TFBGs (i.e., TFBG1, TFBG2) connected to the two switched ports of OSW. The tuning range could cover the whole C-band by switching between the two OSW ports connected to individual TFBG. The original reflected wavelengths of TFBGs are 1540.5 and $1552.68 \mathrm{~nm}$, respectively. Two variable optical attenuators (VOAs) are used in each port for the power equalization.

Figure 4(a) shows signal power versus $1480 \mathrm{~nm}$ pump power for various lengths of EDF. With the selected lasing signal at $1550 \mathrm{~nm}$, the signal power is linearly proportional to the pumping power. The pumping efficiency increases from $3.7 \%$ to $40 \%$ as EDF length increases from $0.8 \mathrm{~m}$ to $5 \mathrm{~m}$. Although the longer EDF length seems to have better conversion efficiency and to generate higher laser power, it also generates more ASE noise which results in lower signal-to-noise-ratio (SNR). As the EDF length increases, the extra gain provided by the pumping power is smaller than the loss attributed by the EDF. For this linear-cavity fiber laser, the parameters for achieving optimum performance are $1.9 \mathrm{~m}$ in length for EDF and 50\% reflectance for the TFBGs.

Figure 4(b) shows the superimposed output spectra of the fiber laser. It is randomly tuned across the C-band with power equalization function. The power equalization can be realized by using VOAs independently. The power uniformity within $\pm 0.1 \mathrm{~dB}$ over the tuning range has been achieved. No polarization mode competition effect is observed partially due to the narrow linewidth of TFBGs. The switching time for OSW is $10 \mathrm{~ms}$ in this case, which depends mainly on the specification of the mechanical OSW.

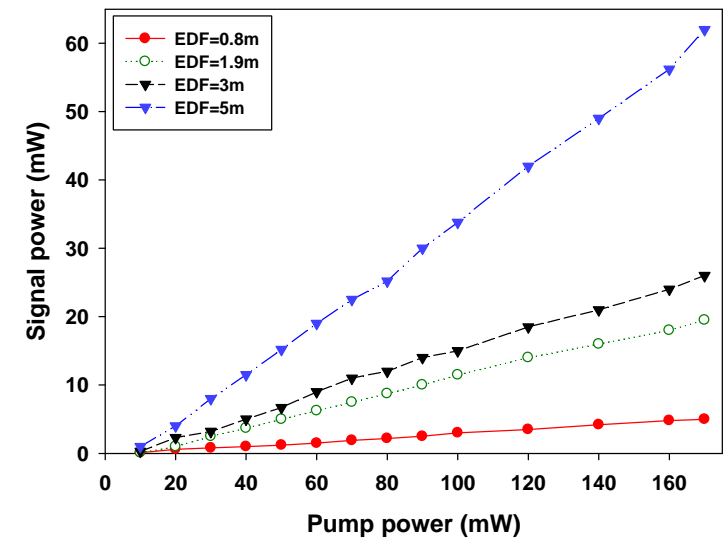

(a)

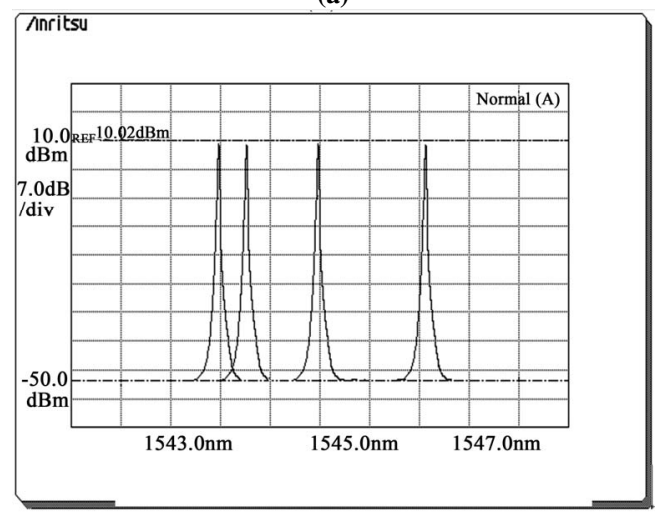

(b)

Figure 4. (a) Laser output power versus pump power using different EDF lengths; (b) superimposed output spectra of tunable fiber laser as wavelength of TFBGs is tuned across C-band after power equalization.

\subsection{Broadband Fiber Mirror as Rear Cavity End}

The proposed BFM-based liner-cavity tunable fiber laser in a backward pump scheme is shown schematically in Figure 5. The laser cavity consists of a BFM, a tunable FBG, and a piece of EDF. The BFM here acts as a broadband wavelength reflector integrated with a TFBG to form a laser cavity. It will lase as long as the reflected wavelength of TFBG is within the reflective range of the BFM. Also, a piece of EDF is inserted into the cavity to act as pump absorber.

Figure 6(a) shows the measured experimental results for the EDF length versus the output power at $1544.8 \mathrm{~nm}$ when Figure 5 has a constant pump power of $150 \mathrm{~mW}$. We find that as the EDF length is increased from 0 to 4 $\mathrm{m}$, the output lasing power is increased accordingly. If the EDF is longer than required, there is a region of the EDF where the pump power is relatively small, the signal has reached saturation intensity, and the gain is decreased according. The curves for various lengths of EDF with threshold power of $8 \mathrm{~mW}$ are shown in Figure 


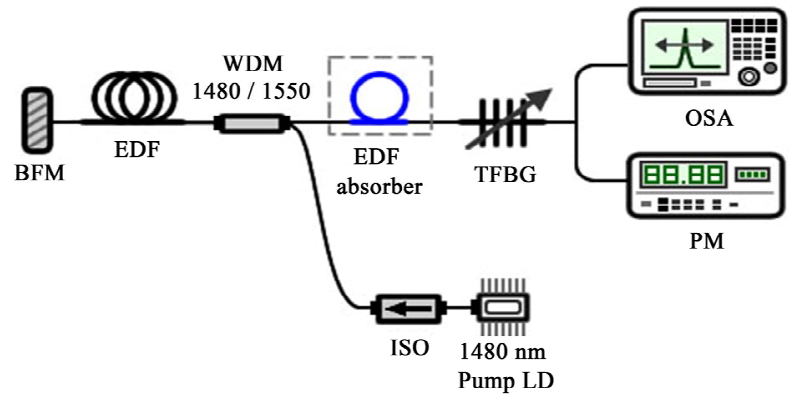

Figure 5. Configuration of proposed BFM-based liner-cavity tunable fiber laser in backward pump scheme with use of residual pump power [11] (PM: power meter).

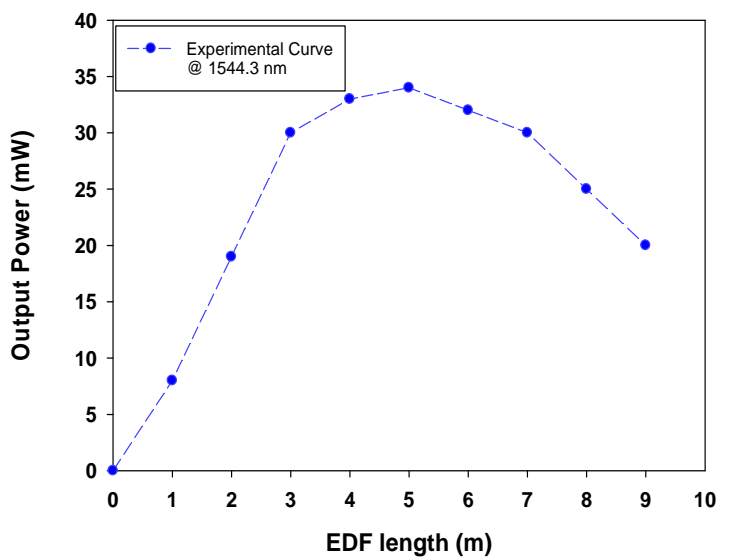

(a)

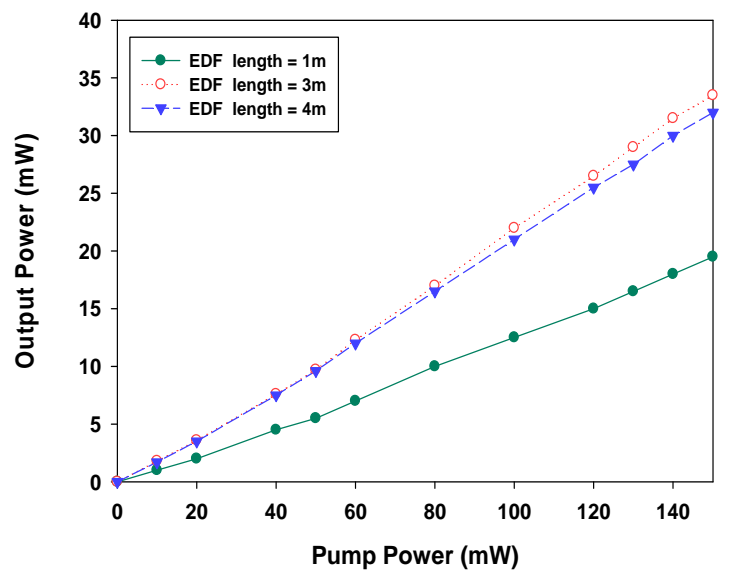

(b)

Figure 6. (a) Experimental results show EDF length against output power as pump power is set at $150 \mathrm{~mW}$; (b) experimental results of pump power versus lasing output power for various lengths of EDF in BFM-based fiber laser scheme.

6(b). The transfer efficiency versus pump power for different lengths of EDF is shown in Figure 7(a). We find that the transfer efficiency is increased as the EDF length increases in the beginning. Then it reaches a constant value of $21.5 \%$ as the pump power is larger than $70 \mathrm{~mW}$.

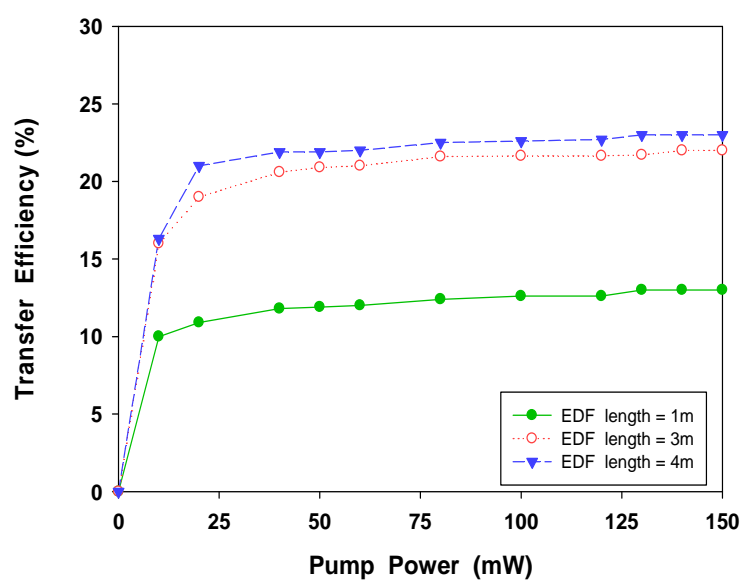

(a)

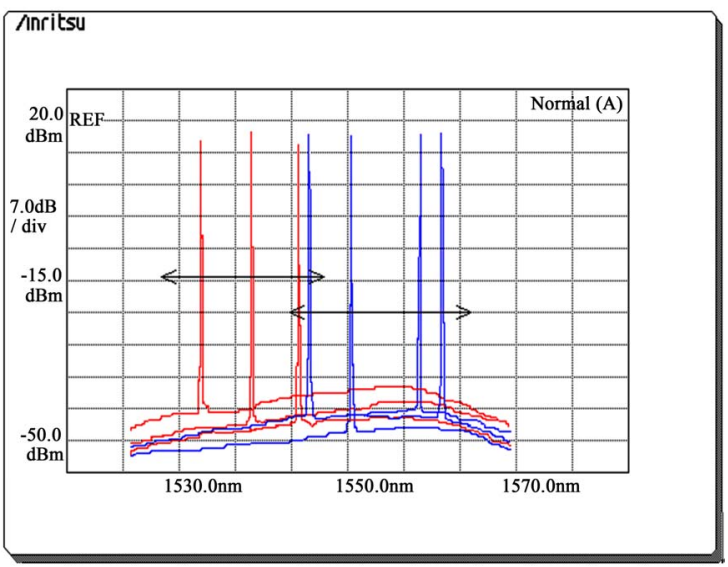

(b)

Figure 7. (a) Experimental results of transfer efficiency versus pump power for different lengths of EDF; (b) superposed output spectra of BFM-based tunable fiber laser. (Wavelength is tuned across C-band using two TFBGs).

The transfer efficiency here is defined as

$$
\eta=\frac{P_{\text {Las }}}{\left(P_{\mathrm{P}}^{\text {in }}-P_{\mathrm{P}}^{\text {th }}\right)}
$$

where $\eta$ is the laser transfer efficiency, $P_{\mathrm{p}}$ in is the input pump power, and $P_{\mathrm{p}}^{\text {th }}$ is the threshold power. Figure 7(b) shows the superimposed output spectra of the tunable fiber laser using two TFBGs with original wavelength of 1539.13 and $1553.0 \mathrm{~nm}$, respectively. High side mode suppression ratio (SMSR) of around $57 \mathrm{~dB}$ was obtained for the entire C-band.

\section{Tunable SWPF-Based Tunable Fiber Lasers: Configurations and Experimental/ Simulation Results}

\subsection{SWPF-Based Tunable Fiber Laser}

In this section, a continuously tunable erbium-doped 
fiber laser is demonstrated by incorporating a tunable SWPF into ring resonator. The wideband tunable SWPF is based on dispersive evanescent tunneling from a sidepolished single-mode fiber and a dispersive optical polymer overlay structure. In fabrication, a portion of the fiber jacket was stripped off and the section was then embedded and glued into the curved V-groove on a silicon substrate, as shown in Figure 8. The central cladding thickness after polishing was around $2.7 \mu \mathrm{m}$. Finally, the characteristics of the well-polished fibers were calibrated by liquid-drop experiments. The effective interaction length was estimated to be $11 \mathrm{~mm}$ at $1550 \mathrm{~nm}$ wavelength for side-polished SMF-28. In Figure 8, the tunable SWPF is incorporated into the resonant cavity to provide a wideband tunable transmission loss window. The dispersive optical polymer overlaying the side-polished fiber is OCK-433 (Nye Lubricants) with the thermo-optic coefficient $\mathrm{d} n_{\mathrm{D}} / \mathrm{d} T$ of $-3.6 \times 10^{-4} /{ }^{\circ} \mathrm{C}$ and is heated by a dual TEC. The refractive index of the OCK-433 decreases with increasing temperature. The tuning efficiency is $7.65 \mathrm{~nm} /{ }^{\circ} \mathrm{C}$ and the signal-to-ASE ratio is around $40 \mathrm{~dB}$. The EDF used here has absorption coefficient of 12 and $30 \mathrm{~dB} / \mathrm{m}$ for 1480 and $1530 \mathrm{~nm}$ wavelength, respectively, and is pumped by a $1480 \mathrm{~nm}$ pump laser diode (LD) in $250 \mathrm{~mW}$ launched power.

To investigate the influences of the sharpness of the spectral cutoff curve, the Cargille liquids were applied on SWPF. The spectral responses of the fiber laser are shown in Figure 9(a). When the refractive indices of $1.456\left(n_{\mathrm{D}}\right)$ and $1.458\left(n_{\mathrm{D}}\right)$ were used, the lasing wavelength moved to shorter wavelengths and the peak power decreased following the gain profile. Subsequently, the Cargille liquids were replaced by OCK-433 and the spectral responses are shown in Figure 9(b). When the temperature cooled down, the lasing wavelengths were moved toward shorter wavelengths again. As the temperature was tuned to $39.6^{\circ} \mathrm{C}$, the lasing wavelength was at $1569.8 \mathrm{~nm}$. Thus, the tuning range of the fiber laser is $26 \mathrm{~nm}$ with temperature variation of $3.4^{\circ} \mathrm{C}$, and typical signal-ASE-ratio is above $40 \mathrm{~dB}$ and the average FWHM is around $0.5 \mathrm{~nm}$.

\subsection{Thermo-Optic Tunable Erbium-Doped FiberRing Laser}

In this subsection, wideband tunable high cutoff-efficiency SWPFs were discretely located in standard silicabased C-band EDF to filter out the $\mathrm{C}+\mathrm{L}$ band ASE so that the optical gain for S-band could be acquired to realize fiber laser. To investigate the amplification characteristics in the S-band, a 980-nm pump laser with 135$\mathrm{mW}$ output power was launched into EDF in a forward pumping scheme. The high-cutoff-efficiency short-pass filters in the 17.5-m-long EDF could discretely suppress the unwanted $\mathrm{C}+\mathrm{L}$ band $\mathrm{ASE}$ and pass the S-band signal

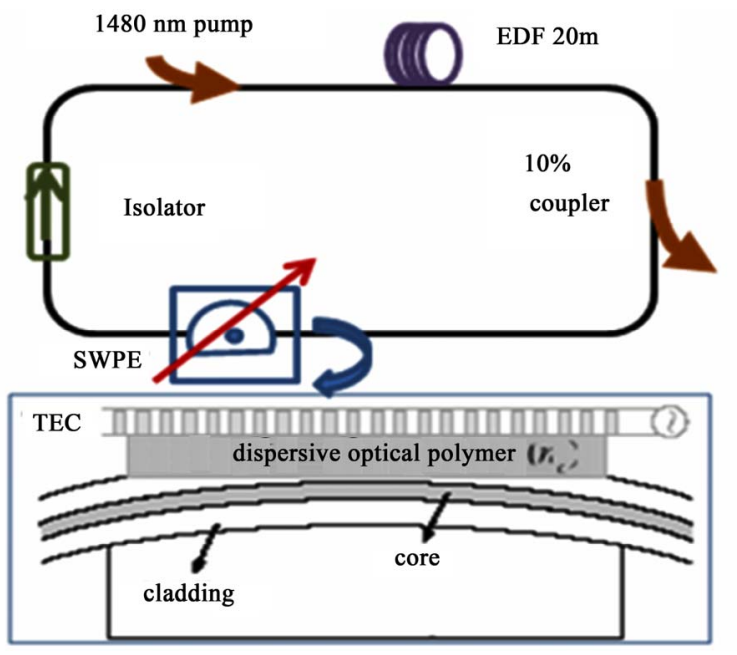

Figure 8. Experimental setup of erbium-doped fiber ring laser using side-polished fiber based tunable SWPF [12].

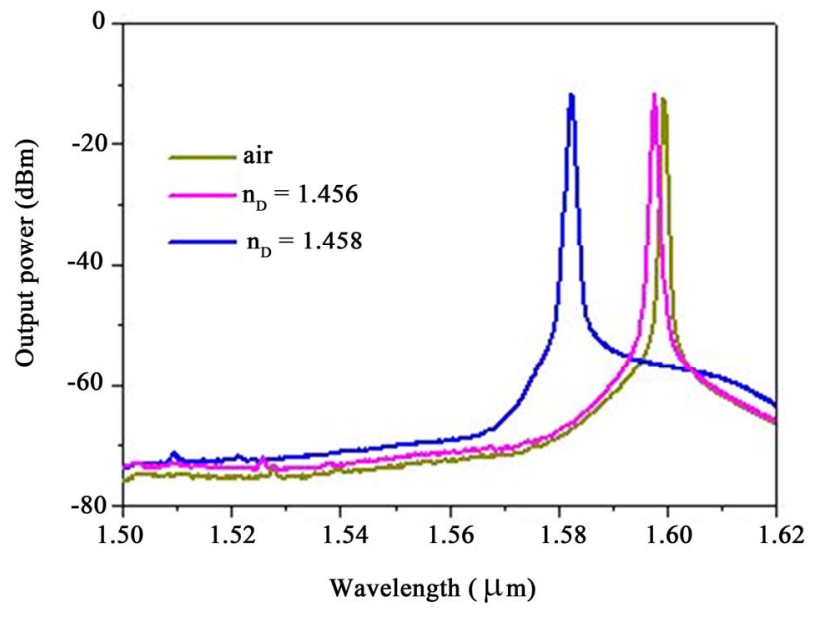

(a)

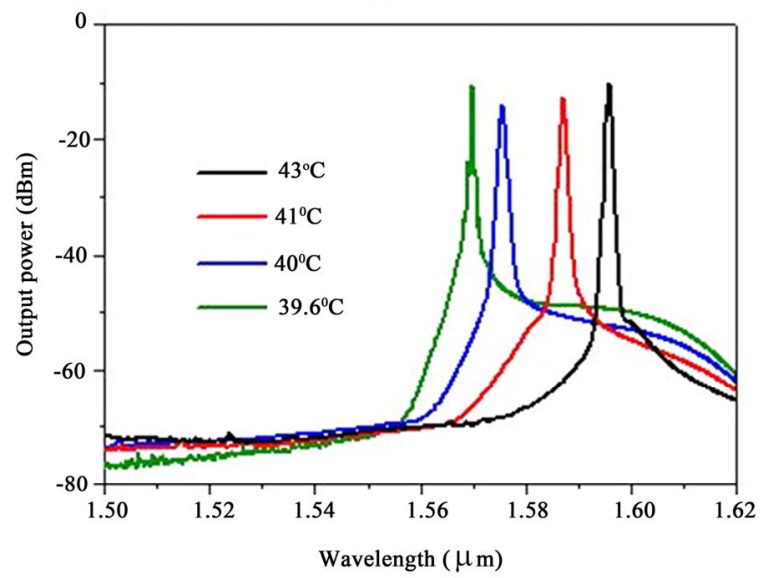

(b)

Figure 9. (a) Spectral responses of EDF fiber ring laser in air and using two kinds of Cargille index liquids on SWPF; (b) spectral responses of wavelength tuning of fiber laser when OCK-433 polymer was cool down [12]. 
and 980-nm pump light. Subsequently, an input power of $-25 \mathrm{dBm}$ was launched into the EDF from distributed feedback laser signals in the S-band. The input signal spectra and amplified output signal spectra in the S-band at $28.6^{\circ} \mathrm{C}$ are shown in Figure 10(a). In the S-band the net signal gain at $1486.9 \mathrm{~nm}$ was measured to be 18.92 dB.

The experimental set-up of the tunable EDF ring laser is shown in Figure 11, where the tunable fused-tapered SWPF with the use of Cargille index liquid $\left(n_{\mathrm{D}}=1.456\right)$ can provide a sharp filter skirt and a deep stop band rejection efficiency ( $>50 \mathrm{~dB}$ ). However, a single local SWPF is inefficient for the standard EDF to be operated at the shorter wavelengths (S-band) of the gain bandwidth. Consequently, we employ four-stage in-line tunable fused-tapered fiber SWPFs discretely located in the standard silica-based EDF to achieve the tunable S-band fiber laser. When the SWPF is turned on, the C + L-band ASE is suppressed to obtain the gain for S-band lasing. The four filters are discretely located in a 16-m-long stan-

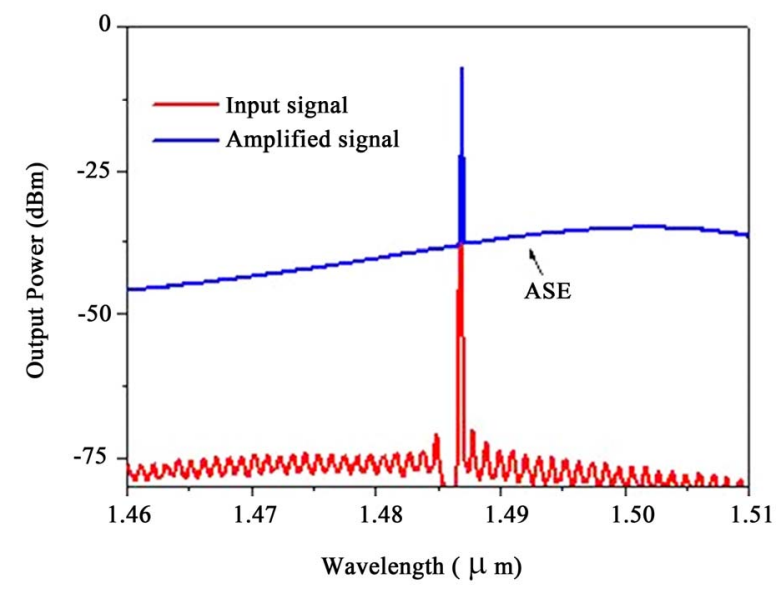

(a)

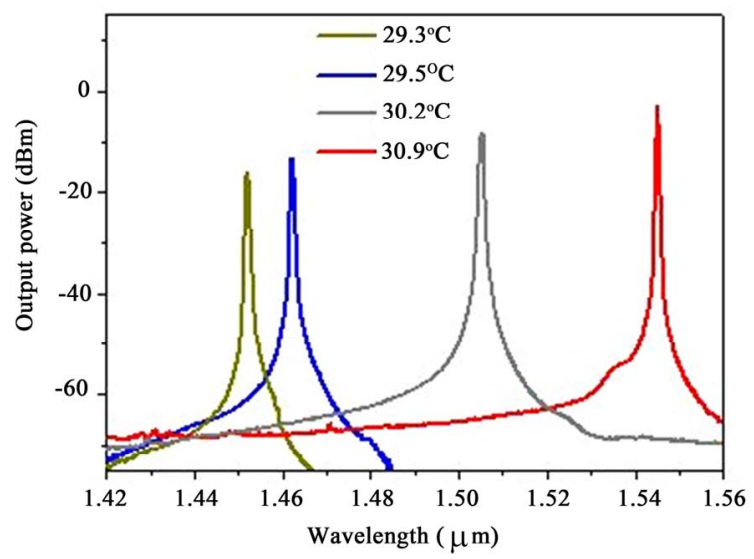

(b)

Figure 10. (a) Amplification spectra of the signals in S-band at $28.6^{\circ} \mathrm{C}$; (b) evolution of output laser spectra by cooling down optical liquid and bending splicing point using the first set of tapered fibers $[13,17]$.

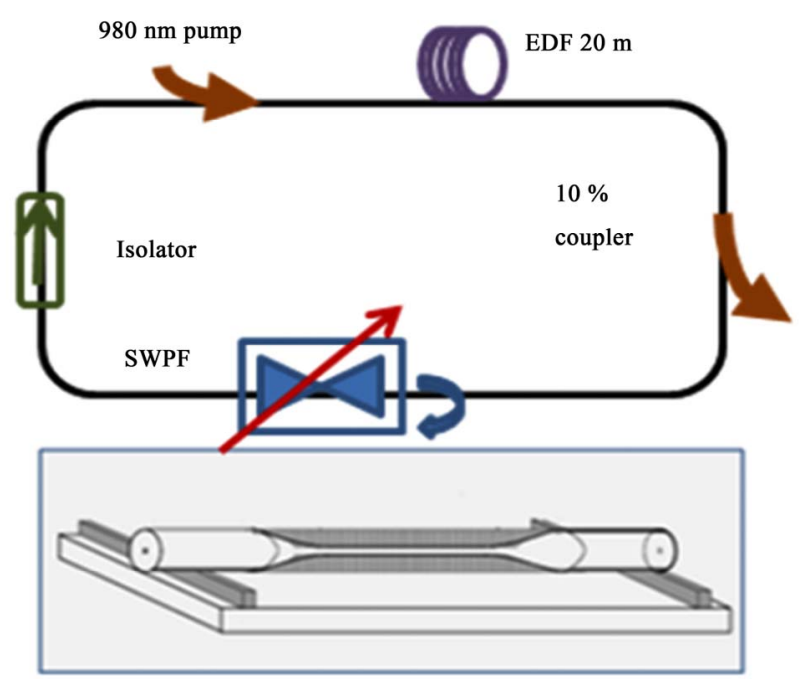

Figure 11. Experimental setup of tunable EDF ring laser towards short-wavelength limit at $1450 \mathrm{~nm}$ (Each 4-m-long EDF and short-pass filter forms a gain stage and there are four gain stages totally in the ring cavity. The FP filter is used for narrowing the laser linewidth down to below 0.2 nm [13]).

dard silica-based C-band EDF to substantially suppress the ASE at the wavelengths longer than the lasing wavelength which can be tuned by varying the applied temperature on SWPFs. When a 980-nm laser with pump power of $208 \mathrm{~mW}$ launches into the EDF, the laser spectra at different temperatures are shown in Figure 10(b). When the applied temperature slightly decreases, the lasing wavelength moves to shorter wavelength. The average $\eta$ for the tunable laser is measured to be as high as $57.3 \mathrm{~nm} /{ }^{\circ} \mathrm{C}$ from 1545.2 to $1451.9 \mathrm{~nm}$ ascribing to the wideband tunable high-cutoff-efficiency SWPFs. The average FWHM is $0.53 \mathrm{~nm}$ and the signal-to-ASE ratio is above $40 \mathrm{~dB}$.

\section{Discussion}

\subsection{Advantages of FBG-Based Tunable Fiber Lasers}

A versatile and cost-effective laser source should have the ability to allow the user to choose which wavelength is needed or the desired scanning range. The wavelength tunable FBG-based lasers we presented here can satisfy such requirement. It is well known that the cavity of a fiber laser may be designed based on a pair of FBGs that work as its end mirrors and determine the resonant wavelength. When one of the resonant wavelengths of the FBGs is changed slightly by tension or heating, the reflection power by the FBG pair at a new laser wavelength will decrease due to wavelength misalignment between them, thus it is difficult to fine-tune the FBG 
pair back to the same wavelength. Nevertheless, either the OC-based or BFM-based laser configuration could overcome such a problem because one FBG only is used to tune the lasing wavelength. Other advantages of FBGbased tunable fiber lasers are: 1) Narrow laser linewidth and near polarization-independent; 2) both the OC- based and BFM-based tunable fiber lasers improve the pumping efficiency by recycling the residual pump power back to the gain medium using backward pumping; 3) the TFBG could be used to tune the desired wavelength precisely and quickly; 4) the proposed FBG-based tunable fiber lasers may use one OSW pair and a plurality of tunable FBGs to expand the output wavelength range; 5) they are simpler and potentially less expensive than other commercial products; and 6) the sizes are compact and the weights are light.

\subsection{Merits of SWPF-Based Tunable Fiber Lasers}

It is advantageous to explore a widely tuning fiber laser with lasing wavelength down to S-band at a high tuning speed. Conventionally, the silica-based EDF at room temperature can only emit fluorescence at wavelengths longer than $1490 \mathrm{~nm}$. Thus, achieving high-performance S-band lasers critically depends on the SWPFs. The sidepolished SWPFs were adopted because they are mechanically strong, and the polishing depth and interaction length can be precisely determined. From a different point of view, SWPFs using the fused-tapering technique are easy, fast, and cost-effective fabrication processes. An optimized side-polishing/tapered fiber filter structure can attain high-cutoff efficiency and wide tuning range. Based on the proposed SWPFs, widely tunable, singlefrequency rare-earth-doped fiber lasers can be achieved. Besides, the SWPF-based tunable fiber lasers have other advantages such as: 1 ) wide tuning range covering the $S$ and C-bands, 2) high power and low noise, 3) simplicity and cost-effectiveness, and 4) high index sensitivity up to $1 \times 10^{-5}$ with high $Q$ resonator.

\subsection{Single-Frequency Design}

To design a single-frequency tunable fiber laser, various kinds of methods such as multiple ring cavities, FBGs, microrings, spatial hole burning in unpumped EDF, and nonlinear loop mirror were proposed. Also, a short cavity length is usually required to enlarge the mode spacing. For the linear-cavity fiber lasers as mentioned, a simpler way to achieve single-longitudinal-mode (SLM) operation is to put a piece of EDF as pump absorber between the WDM coupler and $1 \times 2$ OSW for the OC-based linear-cavity tunable fiber laser as shown in Figure 3; and between the 1480/1550 nm WDM and TFBG for the BFM-based linear-cavity tunable fiber laser as shown in
Figure 5, individually. On the other hand, the SWPF in tunable fiber lasers is naturally a broadband filter that is obviously difficult for single-longitudinal mode laser operation. However, a SWPF made of a highly dispersive waveguide structure can introduce high chromatic dispersion inside the laser cavity to significantly reduce the cavity modes into one. One suggestion is to concatenate the SWPF and an additional ultra-narrowband filter inside the cavity to attain SLM operation.

\section{Conclusions}

Two kinds of tunable fiber-filter-based EDF fiber lasers have been reviewed. Both of them have broadband wavelength tuning range including C-and/or S-band. Using FBG in strain mechanism, we have proposed and demonstrated a tunable FBG-based fiber laser that employs one OC, two homemade TFBGs. The configuration consists of a linear cavity to achieve a wavelength tuning range of $31.5 \mathrm{~nm}$ with $0.05 \mathrm{~nm}$ linewidth and over $60 \mathrm{~dB}$ SNR. The power variation over the entire tuning range is less than $0.1 \mathrm{~dB}$ with power equalization by using low-cost VOAs. Another way is to employ a BFM and tunable FBG at either cavity end of fiber cavity. The BFM acts as a broadband rear-end reflector both for lasing signal and pump source. For wavelength tunable demonstration, power variation over the whole $\mathrm{C}$-band is less than $\pm 1.0 \mathrm{~dB}$ without the usage of power equalization. The time to reach stable laser operation is less than $11 \mathrm{~ms}$ after switching between the two FBGs, and the continuous tuning resolution is less than $0.2 \mathrm{~nm}$ in the whole range. For the SWPF-based tunable fiber laser using temperature tuning mechanism, two tunable SPWFs based erbium-doped fiber lasers were reviewed. The side-polishing and fused-tapering techniques were used to achieve thermo-optic tunable short-wavelengthpass function based on material dispersion discrepancy and variations of waveguide structures. The tuning efficiency is $50 \mathrm{~nm} /{ }^{\circ} \mathrm{C}$, cut-off efficiency is $-1.2 \mathrm{~dB} / \mathrm{nm}$, and rejection efficiency is $55 \mathrm{~dB}$, individually. The widely tunable SWPFs were applied to achieve broadband and high-tuning-efficiency S- and/or C-band EDF ring lasers, which can be tuned close to the short-wavelength edge of gain bandwidth, and the tuning range is $26 \mathrm{~nm}$ with the signal-to-ASE-ratio of around $40 \mathrm{~dB}$, and the FWHM linewidth is about $0.5 \mathrm{~nm}$. All of them have graceful features of simple structure, compactness, ease of connection to fiber components, high-efficiency, and continuous tunability. They are promising for vast applications in lightwave transmission, optical test instrument, fiber-optic gyros, spectroscopy, material processing, fiber sensing, WDM backup light sources, as well as in biophotonics. 


\section{Acknowledgements}

The authors were partially supported by the National Science Council (NSC) (Project Nos. NSC 98-2221-E011-017, NSC 97-2923-E-011-001-MY3, NSC 98-2218 -E-008-004, NSC 98-2221-E-239-001-MY2). We thank Jang W. Y., Wang C. J., Hung K. L., Jhong G. S., Chi S., Tseng S. M., Huang C. M., Lai Y. for discussion, T. Wang and Z. G. Shieh for kind help.

\section{References}

[1] A. Bellemare, J. F. Lemieux, M. Tetu and S. LaRochelle, "Erbium-Doped Ring Lasers Step-Tunable to Exact Multiples of $100 \mathrm{Ghz}$ (ITU-GRID) Using Periodic filter," Proceedings of ECOC'98, Madrid, September 1998, pp. 153-154.

[2] C. S. Kim and J. U. Kang, "Multiwavelength Switching of Raman Fiber Ring Laser Incorporating Composite Polarization-Main Maintaining Fiber Lyot-Sagnac Filter," Applied Optics, Vol. 43, No. 15, 2004, pp. 3151-3157.

[3] C. C. Lee, Y. K. Chen and S. K. Liaw, "Single-Longitudinal-Mode Fiber Laser with Passive Multiple-Ring Cavity and its Application for Video Transmission," Optics Letters, Vol. 23, No. 5, 1998, pp. 358-360.

[4] S. Kim, B. Lee and D. H. Kim, "Experiments on Chaos Synchronization in Two Separate Erbium-Doped Fiber Lasers," IEEE Photonics Technology Letters, Vol. 13, No. 4, 2001, pp. 290-292.

[5] S. Foster, "Spatial Mode Structure of the Distributed Feedback Fiber Laser," IEEE Journal of Quantum Electronics, Vol. 40, No. 7, 2004, pp. 884-892.

[6] M. K. Abd-Rahman and H. Ahmad, "Multiwave-Length Brillouin Erbium Fiber Laser Pumped from FBG Fiber Laser Sharing the Same EDF," Proceedings of the 4th Pacific Rim Conference on Lasers and Electro-Optics, Chiba, July 2001, pp. 40-41.

[7] H. Chen, F. Babin, M. Leblanc and G. W. Schinn, "Widely Tunable Single-Frequency Erbium-Doped Fiber Lasers," IEEE Photonics Technology Letters, Vol. 15, No. 2, 2003, pp. 185-187.

[8] D. Sabourdy, V. Kermene, A. Desfarges-Berthelemot, L. Lefort, A. Barthelemy, P. Even and D. Pureur, "Efficient Coherent Combining of Widely Tunable Fiber Lasers," Optics Express, Vol. 11, No. 2, 2003, pp. 87-97.
[9] Y. C. Zhao, S. Winnall and S. Fleming, "Tunable FiberRing Laser Based on Broad-Band Fiber Bragg Grating and Bending Effects," Microwave and Optical Technology Letters, Vol. 46, No. 6, 2005, pp. 562-563.

[10] S. K. Liaw, W. Y. Jang, C. J. Wang and K. L. Hung, "Pump Efficiency Improvement of a C-Band Tunable Fiber Laser Using Optical Circulator and Tunable Fiber Gratings," Applied Optics, Vol. 46, No. 12, 2007, pp 2280-2285.

[11] S. K. Liaw and G. S. Jhong, "Tunable Fiber Laser Using a Broad-Band Fiber Mirror and a Tunable FBG As Laser-Cavity Ends," IEEE Journal of Quantum Electronics, Vol. 44, No. 6, 2008, pp. 520-527.

[12] N. K. Chen, S. Chi and S. M. Tseng, "An Efficient Local Fundamental-Mode Cutoff for Thermo-Optic Tunable Er3+-Doped Fiber Ring Laser," Optics Express, Vol. 13, No.18, 2005, pp. 7250-7255.

[13] N. K. Chen, C. M. Huang, S. Chi and Y. Lai, "Towards The Short-Wavelength Limit Lasing at $1450 \mathrm{Nm}$ over I-4(13/2)-> I-4(15/2) Transition in Silica-Based ErbiumDoped Fiber," Optics Express, Vol. 15, No. 25, 2007, pp. 16448-16456.

[14] A. D. Kersey, M. A. Davis, H. J. Patrick, M. LeBlanc, K. P. Koo, C. G. Askins, M. A .Putnam and E. J. Friebele, "Fiber Grating Sensors," Journal of Lightwave Technology, Vol. 15, No. 8, 1997, pp. 1442-1463.

[15] M. Arbore, Y. Zhou, H. Thiele, J. Bromage and L. Nelson, "S-Band Erbium-Doped Fiber Amplifiers for WDM Transmission between 1488 and 1508 Nm," Proceedings of Optical Fiber Communication Conference, Georgia, 23-28 March 2003, pp. 374-376.

[16] M. A. Arbore, "Application of Fundamental-Mode Cutoff for Novel Amplifiers and Lasers," Proceedings of Optical Fiber Communication Conference, (OFC 2005), Anaheim, Vol. 5, 6-11 March 2005.

[17] N. K. Chen, K. C. Hsu, S. Chi and Y. Lai, "Tunable Er3+-Doped Fiber Amplifiers Covering S and C + L Bands over 1490-1610 Nm Based on Discrete Fundamental-Mode Cutoff Filters," Optics Letters, Vol. 31, No. 19, 2006, pp. 2842-2844.

[18] S. Y. Chou, K. C. Hsu, N. K. Chen, S. K. Liaw, Y. S. Chih, Y. Lai and S. Chi, "Analysis of Thermo-Optic Tunable Dispersion-Engineered Short-Wavelength-Pass Tapered-Fiber Filters," Journal of Lightwave Technology, Vol. 27, No. 13, 2009, pp. 2208-2215. 